Infektionen

\section{Antibiotikaeinsatz und Resistenzentwicklung bei Neugeborenen weltweit}

Li G et al. Towards Understanding Global Patterns of Antimicrobial Use and Resistance in Neonatal Sepsis: Insights From the NeoAMR Network. Arch Dis Child 2020; 105: 26-31. doi:10.1136/archdischild-2019-316816

Antibiotikaresistenzen (AMR) auf Neugeborenenstationen (NNUs) sind ein signifikantes Problem weltweit und tragen zur erhöhten Neugeborenensterblichkeit bei. In dieser multinationalen Studie wurden die Unterschiede im Hinblick auf Ressourcen, Auswahl der empirischen Antibiotika $(A B)$ und $A M R$ vor allem in Ländern mit niedrigem und mittlerem Einkommen (LMIC) untersucht, um die Beziehung zwischen Mortalität und AMR besser charakterisieren zu können.

Weltweit nimmt die Zahl von ESBL-Infektionen bei Neugeborenen (NG) zu. Eine Reihe von Faktoren kann resistente Infektionen in NG-Einrichtungen beeinflussen. Es wurde vermutet, dass die empirisch eingesetzte $\mathrm{AB}$-Kombination der NG-Sepsis nach der WHO-Guideline in LMICs lokale Resistenzen hervorruft und für die Mortalität verantwortlich ist.

In einer webbasierten Umfrage wurden mithilfe einer REDCap-Datenbank die Daten von 39 NNUs aus 12 Ländern in 4 Kontinenten gesammelt, die am Neonatal AMR Research Network (NeoAMR) teilnehmen. NeoAMR war 2017 mit dem Ziel gegründet worden, neue und global verfügbare empirische $A B$-Strategien für die Therapie der NG-Sepsis im Hinblick auf Resistenzentwicklungen zu etablieren.

Die Umfrage umfasste Fragen zu Finanzierung, Größe und Aufnahmekriterien der NNU, Zugang zu unterstützenden Therapien, antimikrobiellen Richtlinien, Blutkulturisolaten und Resistenzen. Die Daten von jedem stationär aufgenommenen NG wurden anonymisiert auf einem Server der St. George's Universität von London gesammelt.

In die „WHO Essential Medicines List“ (EML) 2017 wurden drei neue Kategorien für $A B$ aufgenommen: „Access“, Watch“ und „Reserve“, die als Reihenfolge zum antimikrobiellen Einsatz für „first-line“, „second-line“ oder "last resort" dienen sollte.

Die Zahl der Lebendgeburten pro Einheit betrug im Zeitraum von 12 Monaten 513-27700 bei einer Bettenzahl von 12-110. Der Anteil der Frühgeborenen $<32$ Schwangerschaftswochen betrug $0-19 \%$.

38 der 39 Abteilungen waren Universitäts- oder Lehrkrankenhäuser, 25 waren öffentlich-, 8 misch- und 6 privat-finanziert. 35 der 39 Abteilungen konnten routinemäßig beatmen, eine Kreislaufunterstützung war in 36, eine parenterale Ernährung in 34 und eine kinderchirurgische Versorgung in 33 Zentren möglich. Die Mehrzahl der Einheiten waren Level 3 nach der Einteilung der British Association of Perinatal Medicine (BAPM). Der Stellenschlüssel betrug im Durchschnitt 1:1 wie von der BAPM empfohlen.

$A B$-Leitlinien für die frühe (EOS) oder späte Sepsis (LOS) waren in 29 von 38 Zentren vorhanden, für die neonatale Meningitis nur in 22 Zentren. 24 Zentren nutzten die WHO-Empfehlung, Penicillin mit einem Aminoglykosid als „first-line“Therapie bei der EOS zu geben. $43 \%$ der empirisch eingesetzten $A B$ entsprachen der Kategorie „Access“, 37\% „Watch“ und $20 \%$ „Reserve“.

31 der 39 Zentren führten Resistenztestungen durch. Unter den Gram-negativen Isolaten fanden sich am meisten Cephalosporin- (26-84\%) und Carbapenem-Resistenzen (0-81\%), unter den Gram-positiven Isolaten Glykopeptid-Resistenzen (0-45\%).

Die Studie hat signifikante Unterschiede hinsichtlich der verfügbaren Ressourcen, der Auswahl der empirischen antimikrobiellen Therapie und der AMR gezeigt. Die Daten bestätigen die Variation der antimikrobiellen Auswahl obwohl es glo- bale Richtlinien gibt. Ungeklärte Fragen betreffen das Verhältnis der positiven Blutkulturen zur Kontaminationsrate und Blutstrominfektion. Auch hängt die neonatale Kolonisation von verschiedenen Faktoren ab, die sich besonders in LMIC während des stationären Aufenthaltes ändern können. Die Vergleichbarkeit ist schwierig, da es keinen internationalen Standard für die Ausbildung in der Neugeborenenpflege gibt.

Die Autoren weisen darauf hin, dass die Sterblichkeit nicht als einziger OutcomeParameter in Betracht gezogen werden sollte, da auch neurologische Beeinträchtigungen als Sepsisfolge vermieden werden sollten.

\section{FAZIT}

In dieser Studie konnte gezeigt werden, welchen Einfluss unterschiedliche organisatorische und therapeutische Maßnahmen auf AMR haben. Diese unterschiedlichen Faktoren gilt es in weiteren Studien zu erfassen, um AMR im Hinblick auf die NG-Mortalität sowie ihre weiteren Auswirkungen zu verhindern. Die Daten zeigen eine hohe Resistenzrate bei den Gram-negativen Keimen. Hier könnte die WHO-Kategorie „Access“, „Watch“, „Reserve“ ein nützlicher Leitfaden zum Monitoring des antimikrobiellen Gebrauchs sein.

Dr. Isabell Hörnig-Franz, Münster 